\title{
Corrigendum: Genetic and Phenotypic Characterization of the Novel Metallo- $\beta$-Lactamase NDM-29 From Escherichia coli
}

\author{
Ying Zhu ${ }^{1,2 \dagger}$, Xinmiao Jia ${ }^{1,3 \dagger}$, Peiyao Jia ${ }^{1,2 \dagger}$, Xue $\mathrm{Li}^{1,4 \dagger}$ and Qiwen Yang ${ }^{1 *}$ \\ ${ }^{1}$ State Key Laboratory of Complex Severe and Rare Diseases, Department of Clinical Laboratory, Peking Union Medical \\ College Hospital, Chinese Academy of Medical Sciences and Peking Union Medical College, Beijing, China, ${ }^{2}$ Graduate \\ School, Peking Union Medical College, Chinese Academy of Medical Sciences, Beijing, China, ${ }^{3}$ State Key Laboratory of \\ Complex Severe and Rare Diseases, Medical Research Center, Peking Union Medical College Hospital, Chinese Academy of \\ Medical Sciences and Peking Union Medical College, Beijing, China, ${ }^{4}$ Department of Clinical Laboratory, Beijing Anzhen \\ Hospital, Capital Medical University, Beijing, China
}

Keywords: carbapenemase, NDM, Escherichia coli, whole-genome sequence, antimicrobial resistance

\section{A Corrigendum on}

Genetic and Phenotypic Characterization of the Novel Metallo- $\beta$-Lactamase NDM-29 From Escherichia coli

by Zhu, Y., Jia, X., Jia, P., Li, X., and Yang, Q. (2021). Front. Microbiol. 12:743981. doi: 10.3389/fmicb.2021.743981

In the original article, Starkova et al. (2021) was not cited in the article. The citation has now been inserted in Introduction, paragraph 2 and Discussion, paragraph 3.

Additionally, there was an error. Our article is the first research on NDM-29 in China rather than in Asia. Corrections have been made to Highlights, paragraph 1; Introduction, paragraph 2; Discussion, paragraph 1; Discussion, paragraph 3; and Discussion, paragraph 4.

The corrected paragraphs appear below.

Highlights, paragraph 1:

"- The first detailed report of the carbapenemase NDM-29 from E. coli and its genetic environment in China.

- A whole genome sequence analysis of the newly found plasmid pNC225-NDM-29 and the gene $b l a_{\mathrm{NDM}-29}$.

- Complete functional verification experiments, including conjugation, transformation, cloning, and fitness cost."

Introduction, paragraph 2:

"In a recent study, we discovered an NDM-29 carbapenemase- producing E. coli strain (19NC225), isolated from a patient's bile in 2019. The gene sequence of bla $a_{\mathrm{NDM}-29}$ from a Klebsiella pneumoniae strain has been submitted to the NCBI database (NCBI), by researchers from Saint Petersburg, Russia (Starkova et al., 2021). Our study is the first detailed report of NDM-29 from E. coli in China, including genetic and phenotypic characterization, and confirms the potential threat of this new NDM-type carbapenemase to be a cause of extensively drug-resistant organism spread." 
Discussion, paragraph 1:

"Here we described a newly found carbapenemase, NDM29 , isolated from a clinical strain of E. coli. The success of conjugation from clinical isolation to $E$. coli EC600, $K$. pneumoniae ATCC 13883, and K. pneumoniae ATCC 700603 indicated the transferability of pNC225-NDM-29. The presence of the ISKpn19-based transposon where the region harboring $b l a_{\mathrm{NDM}-29}$ is located suggested the risk of the spread of resistance caused by NDM-29. Meanwhile, no fitness cost was observed in the transconjugants/transformants containing plasmid pNC225NDM-29, which demonstrated a limited burden from the transferable plasmid. However, the conjugation from donor to recipients (ATCC 13883, ATCC 700603, and DH5 $\alpha$ ) showed low efficiency, which may be due to high energy cost or multibarrier in recipients (Llosa et al., 2002). Although the growth curves did not show a significant impact of transferred plasmid on recipients, a limitation of growth curve that the competitive fitness of donor over recipients was not estimated and needs further study (Hanafi et al., 2016). Anyhow, the dissemination and adaptability of the NDM-29-harboring plasmid among clinical bacteria would be a threat to infectious control."

Discussion, paragraph 3:

"Currently, the mutation of D130G has been reported in NDM- 14, and kinetic analysis indicated that NDM-14

\section{REFERENCES}

Starkova, P., Lazareva, I., Avdeeva, A., Sulian, O., Likholetova, D., Ageevets, V., et al. (2021). Emergence of hybrid resistance and virulence plasmids harboring New Delhi metallo-beta-lactamase in Klebsiella pneumoniae in Russia. Antibiotics 10:691. doi: 10.3390/antibiotics10060691

Publisher's Note: All claims expressed in this article are solely those of the authors and do not necessarily represent those of their affiliated organizations, or those of has greater carbapenem resistance with a higher affinity for imipenem and meropenem (Zou et al., 2015). Based on our research, there is no difference between NDM1 and NDM-29 in terms of susceptibility to carbapenems, which is in accordance with the findings of Starkova et al. (2021)."

Discussion, paragraph 4:

"In conclusion, we report the identification of a novel class B enzyme with carbapenemase activity, NDM-29, in a clinical E. coli isolate. The novel $b l a_{\mathrm{NDM}-29}$ is first detected in China and was obtained from a MDR E. coli strain isolated from bile of a patient with biliary tract infection. The strain, containing two plasmids (pNC225-TEM1B and pNC225-NDM-29), belongs to ST1485 and O83:H42, showed homology with E. coli MS6198 from Australian (Hancock et al., 2017), which harbors bla $a_{\mathrm{NDM}-1}$. The plasmid, pNC225NDM-29, which encods $b l a_{\mathrm{NDM}-29}$, exhibited 99\% identity with six bla $a_{\mathrm{NDM}-1}$-carrying plasmids, especially a IncN1 plasmid pNDM-BTR from an E. coli in urine specimen $(99.96 \%$ identity and $100 \%$ coverage), and showed responsibility for the MDR phenotype."

The authors apologize for these errors and state that this does not change the scientific conclusions of the article in any way. The original article has been updated. the publisher, the editors and the reviewers. Any product that may be evaluated in this article, or claim that may be made by its manufacturer, is not guaranteed or endorsed by the publisher.

Copyright $(2021 \mathrm{Zhu}$, Jia, Jia, Li and Yang. This is an open-access article distributed under the terms of the Creative Commons Attribution License (CC BY). The use, distribution or reproduction in other forums is permitted, provided the original author(s) and the copyright owner(s) are credited and that the original publication in this journal is cited, in accordance with accepted academic practice. No use, distribution or reproduction is permitted which does not comply with these terms. 